\title{
A New Combination of RAKE Receiver and Adaptive Antenna Array Beamformer for Multiuser Detection in WCDMA Systems
}

\author{
Shahriar Shirvani-Moghaddam ${ }^{1}$ and Hajar Sadeghi² \\ ${ }^{1}$ Digital Communications Signal Processing (DCSP) Research Lab., Faculty of Electrical and Computer Engineering, \\ Shahid Rajaee Teacher Training University (SRTTU), 16788-15811 Tehran, Iran \\ ${ }^{2}$ Department of Electrical Engineering, Islamic Azad University, Tehran South Branch, 177761365 Tehran, Iran
}

Correspondence should be addressed to Shahriar Shirvani-Moghaddam,sh_shirvani@srttu.edu

Received 8 July 2011; Revised 22 August 2011; Accepted 23 August 2011

Academic Editor: Mandeep Jit Singh

Copyright ( $(2011$ S. Shirvani-Moghaddam and H. Sadeghi. This is an open access article distributed under the Creative Commons Attribution License, which permits unrestricted use, distribution, and reproduction in any medium, provided the original work is properly cited.

The aim of this paper is to combine smart antenna beamforming and RAKE receiver in wideband code division multiple access (WCDMA). The proposed method combines spatial diversity as well as temporal diversity to improve the performance and overcome both interferences and multipath fading. This investigation has focused on one of the new proposed blind beamforming algorithms. It is based on constrained constant modulus (CCM) algorithm which is used for deriving a recursive-least-squares (RLS-) type optimization algorithm. We illustrate the comparison of bit error rate (BER) of the proposed receiver with simple correlator and also 1D-RAKE receiver in multiuser detection (MUD) WCDMA. The simulation results show that the proposed 2D-RAKE receiver offers lower BER rather than conventional ones, that is, it is an effective solution for decreasing the effect of interference and increasing the capacity, in a joint state.

\section{Introduction}

In the cellular mobile communications, increasing demand to accommodate more users and new high bit rate data services becomes more and more obvious. Therefore thirdgeneration $(3 \mathrm{G})$ communications are oriented towards multimedia message capability [1] rather than voice communications. Wideband code division multiple access (WCDMA) has been adopted as the air-interface technology by the $3 \mathrm{G}$ wireless communication systems [2]. WCDMA is the wideband direct-sequence CDMA (DS-CDMA) system, that is, user information bits are spread over a wide bandwidth by multiplying the user data with pseudorandom bits (called chips). Some of the most important features of WCDMA are providing high capacities such as transmitting variable data rates with different mobility and quality of service requirements (multimedia services) and reducing the transmitted power [1]. In this system, receiver is a simple correlator which is not optimal due to its disability for combating the multipath effects. RAKE combiner is a popular and effective receiver utilizing temporal diversity in the presence of multipath effects. But unfortunately its performance is limited by multiaccess interference (MAI) because of disability for separating additive white Gaussian noise (AWGN) from MAI [3-7].

Since in WCDMA system, all of the users share the same frequency through the mobile radio channel, any other active users are cochannel interference to the desired user. Indeed, the system performance in WCDMA is limited by interference. Also one of the worse disadvantages of WCDMA is near-far effect. Recently, different ways have been presented to solve these problems. Some research works focused on introducing and using better receivers and other ones offer combined antenna beamforming and WCDMA receiver.

Reference [6] develops an adaptive G-RAKE receiver, employing practical algorithms for finger placement and weight computation. It provides significant gains in performance by suppressing interference. In [8] a RAKE receiver has been proposed which works on chip level equalization on each RAKE finger to cancel MAI in multipath channel. 
Adaptive antenna array systems combine an antenna array with a digital signal processing (DSP) unit. Using the estimated angle of signals arrival, this system form the main lobe of the antenna toward the desired signal and nulls on the direction of interferences. It enhances the quality of received desired signal, suppresses all interfering signals, increases spectrum efficiency, extends range coverage, and enlarges capacity $[8,9]$. Recently, different research works have been presented which combine antenna beamforming and RAKE receiver for WCDMA applications. Due to the main goal of this paper, some of them are mentioned below.

Reference [9] uses conjugate gradient (CG) adaptive beamforming for space diversity processing in each RAKE finger in a DS-CDMA. This paper improves network bit error rate (BER) and capacity of the system, using base-station assignment method based on minimizing the transmitter power (BSA-MTP) technique and power control error (PCE) in comparison with the conventional case, and adaptive beamforming passes the desired users' signal in an arbitrary path. Also it reduces the MAI from other users and cancels the interpath interference (IPI) in other paths in each RAKE finger.

Reference [10] develops a new scheme of blind beamformer for each branch of RAKE receiver [11] to suppress MAI, jamming, and multipath before combining. It shows better performance than the pre- and postcorrelation (PAPC) and the filter pair (FP) schemes.

In [12] fixed-beam antenna array beamforming is combined with new improved version of root multiple signal classification (Root-MUSIC), direction of arrival (DOA) estimation algorithm. In this way, it works as a spatial filter to reduce the cochannel interference and noise.

A beamformer-RAKE receiver is a computationally feasible solution to allow temporal-spatial signal processing for effectively overcoming signal fading and MAI. In [13], the performance of one type of beamformer-RAKE receiver in the geometrically based single bounce (GBSB) circular channel with the uplink WCDMA signal standard has been investigated. The least mean squares (LMS) and the recursive least squares (RLS) beamforming techniques and also maximal ratio combining (MRC) coherent RAKE receiver with different number of fingers are considered for simulation. The performances of the aforementioned beamforming techniques are compared in terms of BER against the number of users in different antenna elements and RAKE fingers.

In [14-18] adding spatial processing achieved by digital beamforming antenna array systems to the RAKE receiver is proposed. This idea is one of the most promising techniques for increasing capacity, radio resource management (RRM), canceling the interferences such as MAI, and near-far effect in WCDMA systems.

In [19], a code-CCM design criterion for linear receivers of DS-CDMA is investigated in multipath channels. In this research, a computationally efficient RLS-type algorithm for jointly estimating the parameters of the channel and the receiver is developed in order to suppress MAI and intersymbol interference (ISI).

In [20], a constant modulus algorithm-based multi user detection (MUD) scheme is proposed for a communication system under multipath propagation. To mitigate channel distortion and multiuser interference, authors integrated multiple constraints into the optimization criterion. The results of this investigation show that the ability of the detector to remove all interferences is ensured in the absence of noise when the constraints are properly preselected. However, in the presence of noise, the constraints highly affect the performance of the receiver. In order to optimally combine signals from different paths to achieve performance gains, those constraints can also be treated as variables and jointly optimized with the receiver, as verified by numerical examples.

According to the above-mentioned study, the main goal of this paper is to combine RAKE receiver (temporal diversity) of WCDMA system with smart antenna (spatial diversity), to reduce the effect of MAI in high data rates, and to increase the quality of service and/or capacity. In this research constrained constant modulus-recursive least squares (CCM-RLS), as mentioned in [21], is considered as proper beamforming algorithm. In this investigation, the number of antenna array beamformers is the same as number of users.

The rest of this paper is organized as follows. Section 2 introduces system model which contains the basics of WCDMA system, its receivers, antenna array, and its algorithm. The new proposed receiver including antenna beamformer and RAKE receiver is presented in Section 3. Section 4 shows the simulation results, and finally Section 5 concludes this investigation.

Notation. $(\cdot)^{H}$ is reserved for the matrix Hermitian, $(\cdot)^{T}$ for the matrix transpose, $(\cdot)^{-1}$ for the matrix inverse, $(\cdot)^{*}$ for the complex conjugate, and $I_{m \times m}$ denotes the $m \times m$ identity matrix.

\section{System Model}

2.1. Direct-Sequence Code-Division Multiple Access. A simplified model for a received signal in a baseband DS-CDMA system is as

$$
x(t)=\sum_{q=1}^{Q} x_{q}(t)+n(t)
$$

where $x_{q}(t)$ is the received signal contribution from the $q$ th user, $Q$ is the number of users in the same channel, and $n(t)$ is the AWGN. $x_{q}(t)$ can be expressed as:

$$
x_{q}(t)=\sum_{k=-\infty}^{+\infty} s_{q}(t) \cdot\left[h_{q}(t-k T) * C_{q}(t-k T)\right],
$$

where $\left\{s_{q}\right\}$ is the data bit stream $\left(s_{q}(k)= \pm 1\right), T$ is the period of the data symbol and the channel given by convolution between $h_{q}(\cdot)$, the channel impulse response, and $C_{q}(\cdot)$, the $q$ th user's spreading waveform:

$$
C_{q}(t)=\sum_{n=0}^{\mathrm{SF}-1} c_{q}(n) \cdot g\left(t-n T_{c}\right)
$$


A unique pseudorandom noise (PN) code sequence $c_{q}(n), n=0, \ldots, \mathrm{SF}$ is assigned to user $q \cdot g(\cdot)$ is the chip pulse waveform, and $T_{c}=T / S F$, where SF is spreading factor and $T_{c}$ is the chip period. The most common PN code families for spreading signal are Walsh-Hadamard codes that are orthogonal to overcome user interferences on each other $[1,3,22]$, that is,

$$
\sum_{n=1}^{\mathrm{SF}} c_{i}(n) c_{j}(n)=0, \quad \forall i \neq j
$$

WCDMA is a kind of DS-CDMA including higher channel bandwidth and chip rate. Since WCDMA is accepted for 3G wireless, this investigation focused on its uplink and Walsh code properties in one cell.

2.2. Simple Correlator. This receiver first multiplies the received signal into the spreading code $c_{q}(\cdot)$ to detect each user and then integrate it. The correlator output is given by

$$
r_{q}(k)=\int_{k T}^{(k+1) T} x(t) \cdot c_{q}(t-k T) d t .
$$

The simple correlator will be optimal if the following conditions are met.

(1) The codes will be orthonormal (orthogonal and normalized, in a joint state), that is,

$$
\int_{0}^{T} c_{i}(t) \cdot c_{j}(t) \cdot d t= \begin{cases}0, & \forall i \neq j \\ 1, & \forall i=j .\end{cases}
$$

(2) Perfect symbol synchronization is achieved.

(3) Channel contains AWGN without any multipath.

2.3. 1D-RAKE. A famous receiver in the presence of multipath is the RAKE receiver, first proposed by Price and Green in 1958 [23]. The RAKE receiver uses multiple correlators using one finger for each path, which it is assumed that the time delay of each multipath is determined. Therefore this receiver overcomes multipath effects. Then all the multipath components will be combined to obtain the received signal with improved quality. There are different types of combining schemes that in this investigation equal gain combining (EGC) is used, that is, $\alpha_{i}{ }^{*}=1$. This receiver uses temporal diversity and maximizes the signal to noise ratio (SNR). For combating multipath and multi user interferences the channel should be estimated well.

Figure 1 illustrates RAKE receiver. The RAKE output is as follows:

$$
r_{q}(k)=\sum_{l=1}^{L} \alpha_{l q} * \cdot\left[\int_{k T}^{(k+1) T} x(t) \cdot c_{q}(t-k T) d t\right] .
$$

One of the weaknesses of RAKE receiver is the complexity when the number of correlators increases. Unlike the goal it raises the power and cost $[3,4,24,25]$.

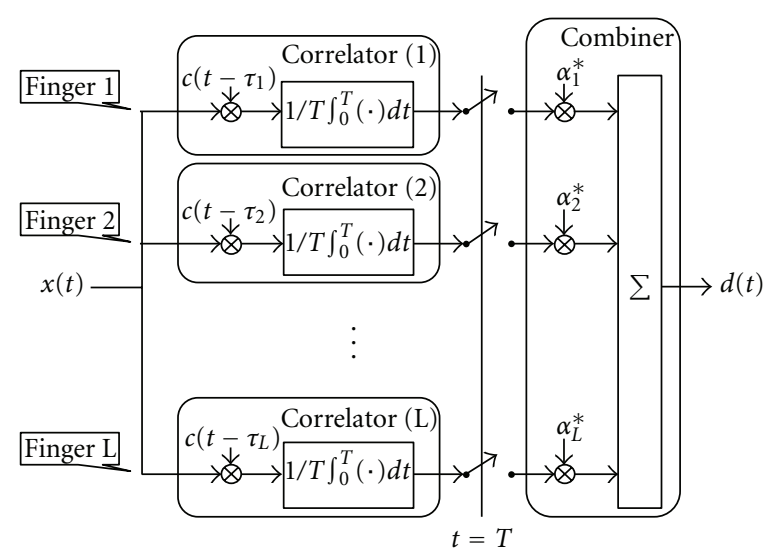

FIGURE 1: 1D-RAKE receiver [3].

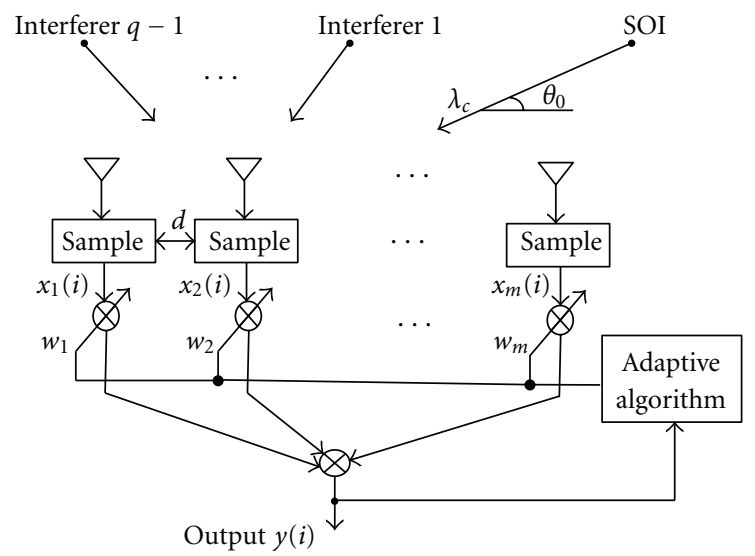

Figure 2: Adaptive beamforming [28].

2.4. Adaptive Antenna Array. Figure 2 shows the adaptive array antenna beamforming or smart antenna scheme. It is supposed that $q$ users signals impinge on the uniform linear array (ULA) of $m(m \geq q)$ sensor elements from sources with DOAs $\theta=\left[\theta_{0}, \ldots, \theta_{q-1}\right]^{T} \in c^{q \times 1}$. It is also assumed that the propagating signals in transmitter and receiver are produced by point sources which are in the far field, namely, that is why the spherically propagating wave can be approximated using a plane wave [26].

The vector of the $i$ th received signal of the array sensors can be modeled as [27]

$$
\begin{gathered}
x(i)=A(\theta) \cdot s(i)+n(i), \quad i=1, \ldots, m, \\
A(\theta)=\left[a\left(\theta_{0}\right), \ldots, a\left(\theta_{q-1}\right)\right] \in c^{m \times q}, \\
a\left(\theta_{k}\right)=\left[1, e^{-2 \pi j(d / \lambda) \cos \theta_{k}}, \ldots, e^{-2 \pi j(m-1)(d / \lambda) \cos \theta_{k}}\right]^{T}, \\
k=0, \ldots, q-1,
\end{gathered}
$$

where $A(\theta)$ is a complex matrix including the steering vectors $a\left(\theta_{k}\right) \in c^{m \times 1}, d=\lambda / 2$ is the interelement distance of the array, $\lambda$ is the wavelength, $s(i) \in R^{q \times 1}$ is a data vector and it 


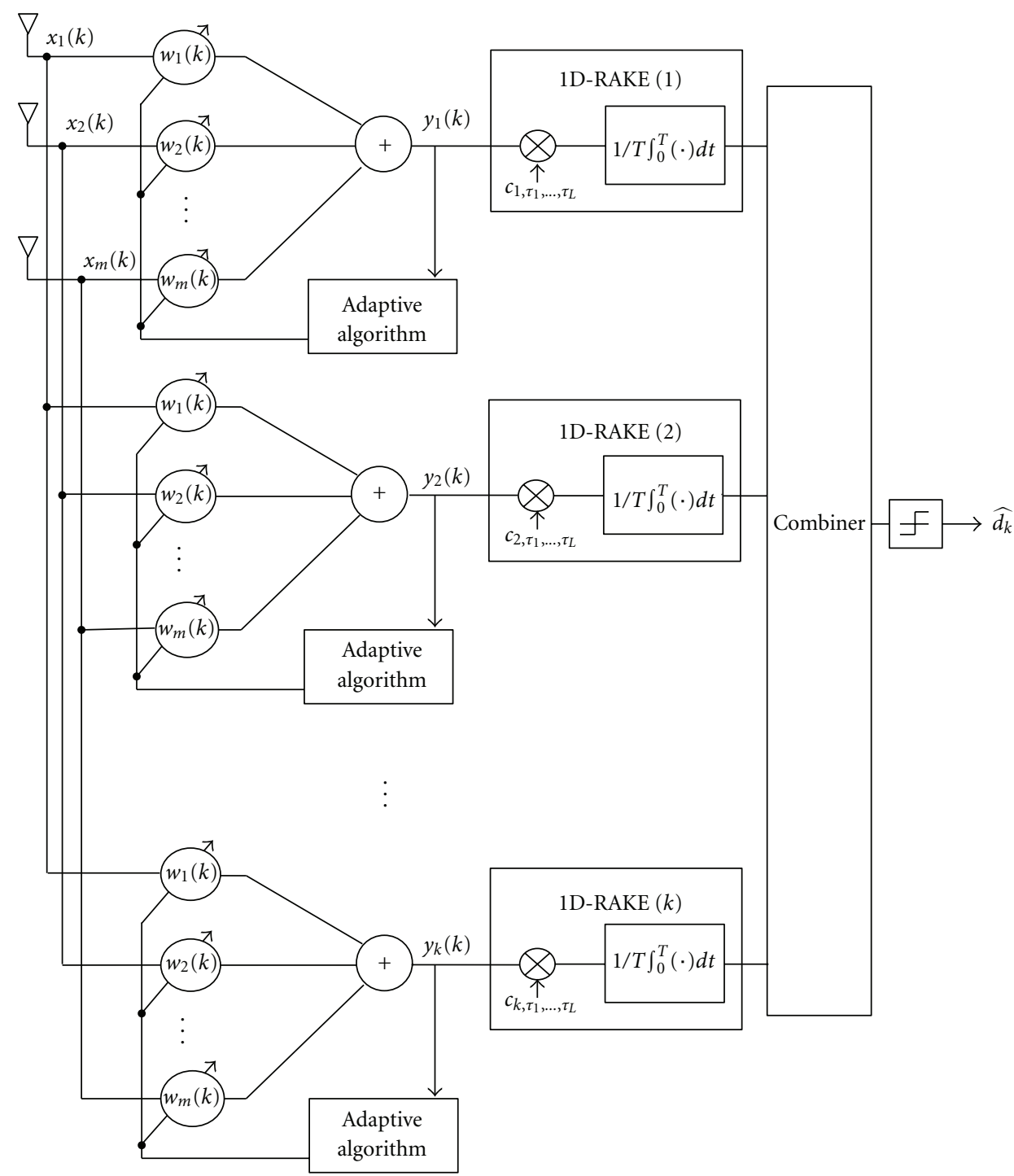

FIGURE 3: WCDMA system using a combination of 2D-RAKE receiver and CCM-RLS beamforming.

is considered to be uncorrelated, $n(i) \in c^{m \times 1}$ is the complex vector of AWGN with zero mean, and $N$ is the number of snapshots.

The output of a narrowband beamformer is given by

$$
y(i)=w^{H} \cdot x(i)
$$

where $w=\left[w_{1}, \ldots, w_{m}\right]^{T} \in c^{m \times 1}$ is the complex weight vector.

\section{Proposed Receiver}

This investigation focused on adaptive base-station antenna beamforming for WCDMA systems in multi user applications (MUAs). The purpose of it is to combine a $1 \mathrm{D}$ RAKE receiver with a blind constant modulus beamforming adaptive array antenna for each user. CCM-RLS algorithm is selected in this research. This algorithm was proposed by Wang and de Lamare [28], for blind adaptive beamforming.
This scheme improves a cost function of constant modulus criterion for the array weight adaptation, using an RLS-type algorithm. In the CCM-RLS algorithm, weights should be updated as

$$
w(i)=\left[a^{H}\left(\theta_{0}\right) \cdot P(i) \cdot a\left(\theta_{0}\right)\right]^{-1} P(i) \cdot a\left(\theta_{0}\right),
$$

where $P(i)=R^{-1}(i)$ is given by

$$
\begin{gathered}
P(i)=\alpha^{-1} P(i-1)-\alpha^{-1} \cdot k(i) \cdot x^{H}(i) \cdot P(i-1), \\
k(i)=\frac{\pi(i)}{(\alpha / 2 e(i))+x^{H}(i) \cdot \pi(i)}, \\
\pi(i)=P(i-1) \cdot x(i), \\
e(i)=\left[\left|w^{H}(i) \cdot x(i)\right|^{2}-1\right],
\end{gathered}
$$

where $w(0)=0, p(i)=\delta^{-1} I_{m \times m}, \delta$ is a positive real number called the regularization parameter that it is small 


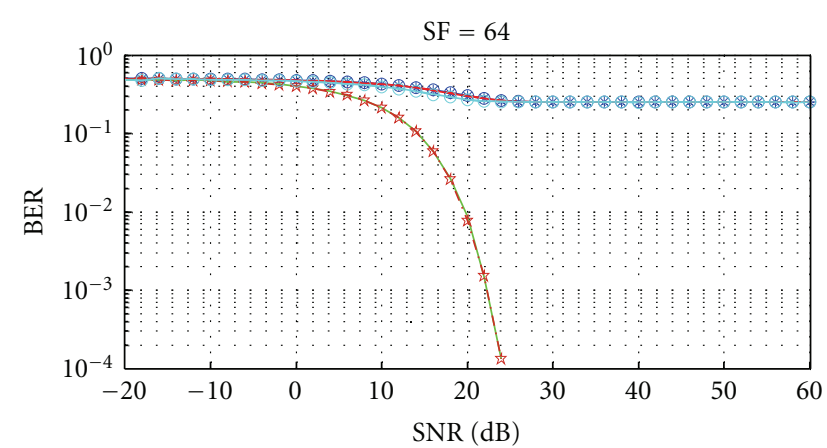

$\begin{array}{ll}- \text { Conventional-user1 } & - \text { 2D RAKE + CCM-RLS BF-user1 } \\ -*-\text { Conventional-user2 } & - \text { 2D RAKE + CCM-RLS BF-user2 } \\ - \text { 1D RAKE-user1 } & \text { Conventional + CCM-RLS BF-user1 } \\ -- \text { 1D RAKE-user2 } & - \text { Conventional + CCM-RLS BF-user2 }\end{array}$

(a)

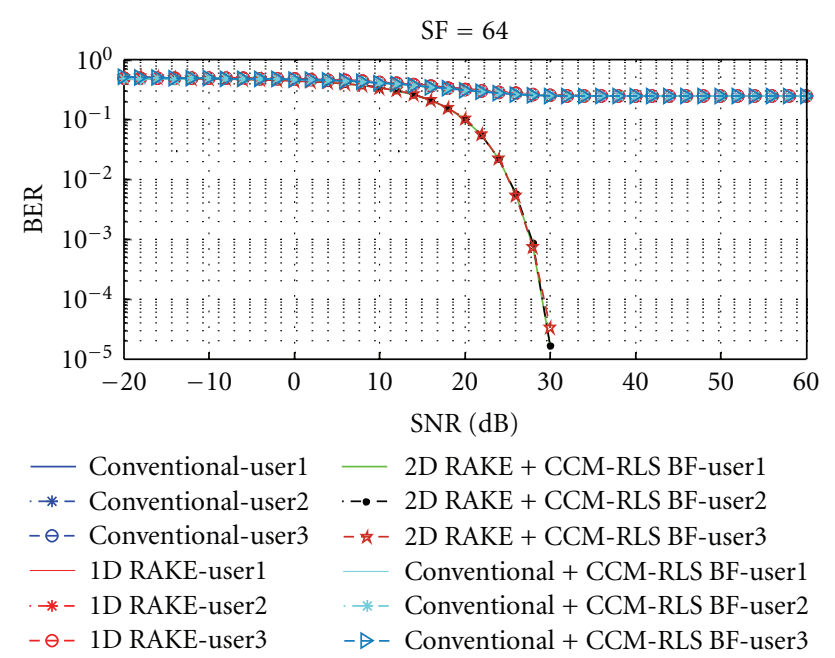

(b)

FIgURE 4: Performance of different conventional and proposed WCDMA receivers considering 4-element antenna array in AWGN channel for (a) 2 users, (b) 3 users.

for high SNRs and vice versa, $I$ is the $m \times m$ identity matrix and $\alpha$ is a forgetting factor close to, but less than, unity. According to $[29,30]$ although CCM-RLS beamforming algorithm has more complexity than CCM-SG, CCM-MASS, and CCM-TASS, due to its fast convergence, there are no local minima, high signal to interference plus noise ratio (SINR) and acceptable BER; in this paper it is selected for multi-user detection in WCDMA system.

This is clearly shown in Figure 3. Thereby, using both diversity, temporal as well as spatial, it makes better detection, reduces multiple access interference in the high data rates, and increases capacity and/or quality in other words.

\section{Simulation Results}

In this section, the effectiveness of the proposed receiver in comparison with the conventional, 1D-RAKE and also correlator receiver plus CCM-RLS antenna array beamformer

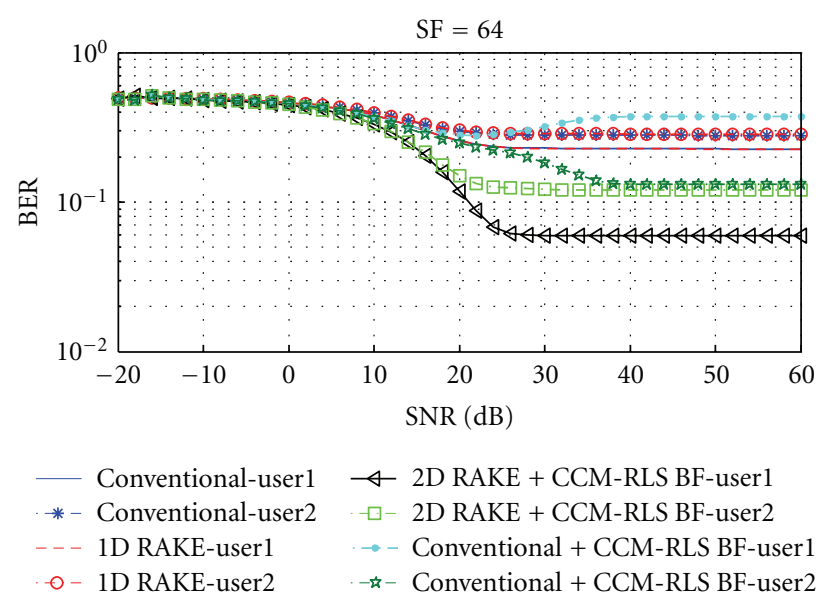

(a)
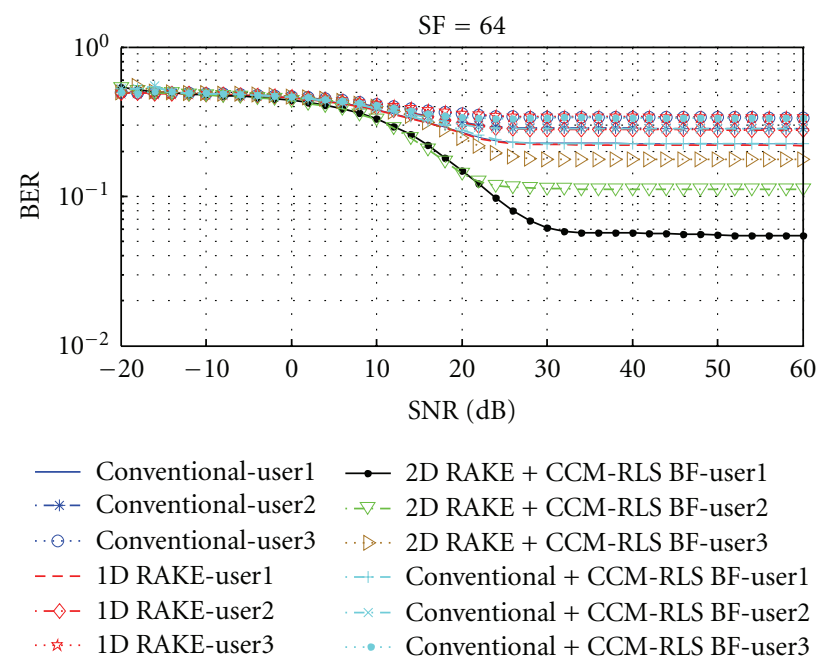

(b)

Figure 5: Performance of different conventional and proposed WCDMA receivers considering 4-element antenna array in 2-path AWGN fading channel for (a) 2 users, (b) 3 users.

is investigated, numerically. The received signal model for the uplink of a generic WCDMA system with short codes is considered. The system chip rate is $3.84 \mathrm{Mcps}$ in $5 \mathrm{MHz}$ channel bandwidth.

Simulations are carried out under stationary scenarios, and there is perfect synchronization between transmitter and receiver. The simulated multiuser environment consists of up to 5 active users with unitary power which means that there is a strict power control in the system. The users are spread with orthogonal Walsh codes of length SF $=64$. The BER of four types of receivers in both AWGN channel and fast fading AWGN channel including up to 3 multipaths for each user is evaluated in simulation where $0 \leq \tau<\mathrm{SF} \cdot T_{c}$. In all experiments the attenuation coefficients in channel are uniform distributed between $0.8-1$. In simulations, it is assumed that the channel is perfectly estimated and the DOA of sources is estimated before the blind beamformer. It is assumed that the number of ULA elements is 4, 

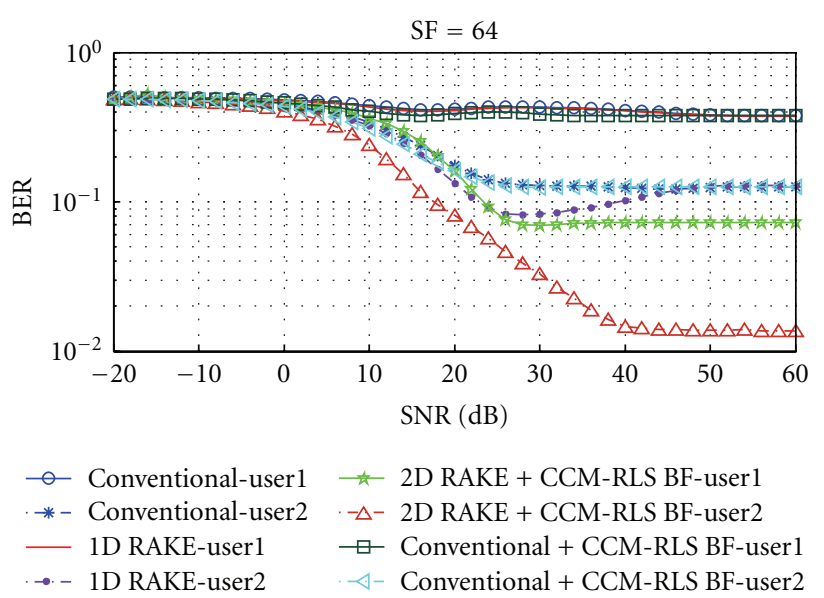

(a)

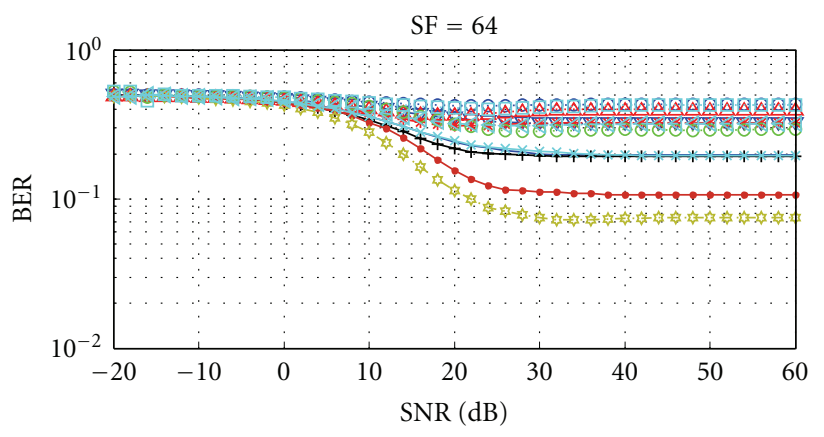

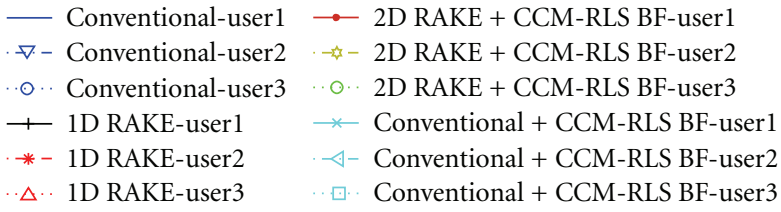

(b)

Figure 6: Performance of different conventional and proposed WCDMA receivers considering 4-element antenna array in 3-path AWGN fading channel for (a) 2 users, (b) 3 users.

except in Figures 8 and 9 which show the effect of array elements. In each transmitter, binary phase shift keying (BPSK) modulation method is employed. Each simulated point in different curves is obtained by 120 iterations. To initiate the CCM-RLS algorithm it is assumed that $w(0)=$ $0, p(0)=\delta^{-1} \cdot I_{m \times m}, \delta=10, \alpha=0.998$.

Figure 4 compares the performance of the four abovementioned receivers in AWGN channel with (a) two and (b) three users. Since there is not any multipath, as expected, conventional receiver and RAKE have the same performance. It is obvious because in this condition they have similar structure. The conventional receiver + CCMRLS beamformer performs better, in $\mathrm{SNR}<30 \mathrm{~dB}$. It means that this method is not an appropriate one because it creates complexity but there is no improvement in the results. In contrast, 2D-RAKE plus CCM-RLS beamforming decreases
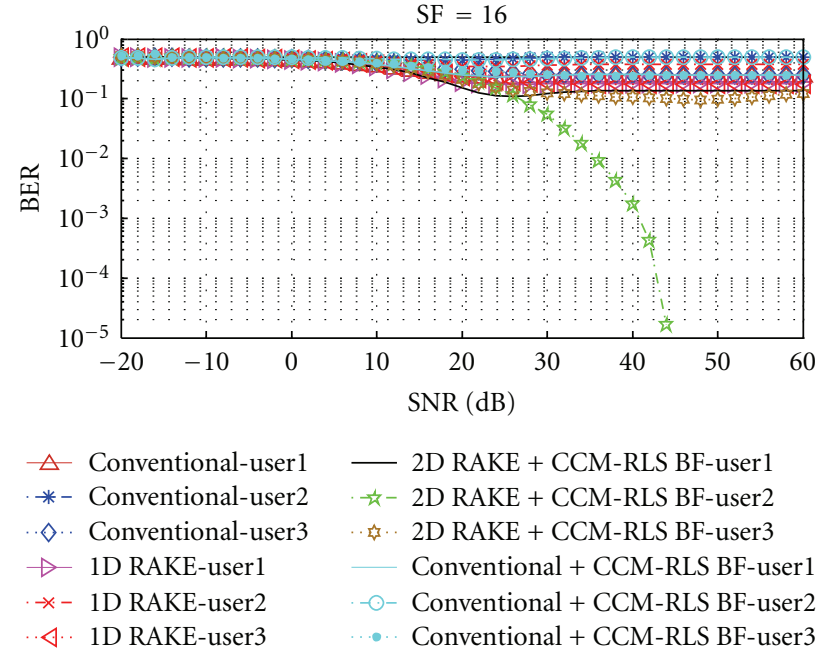

(a)

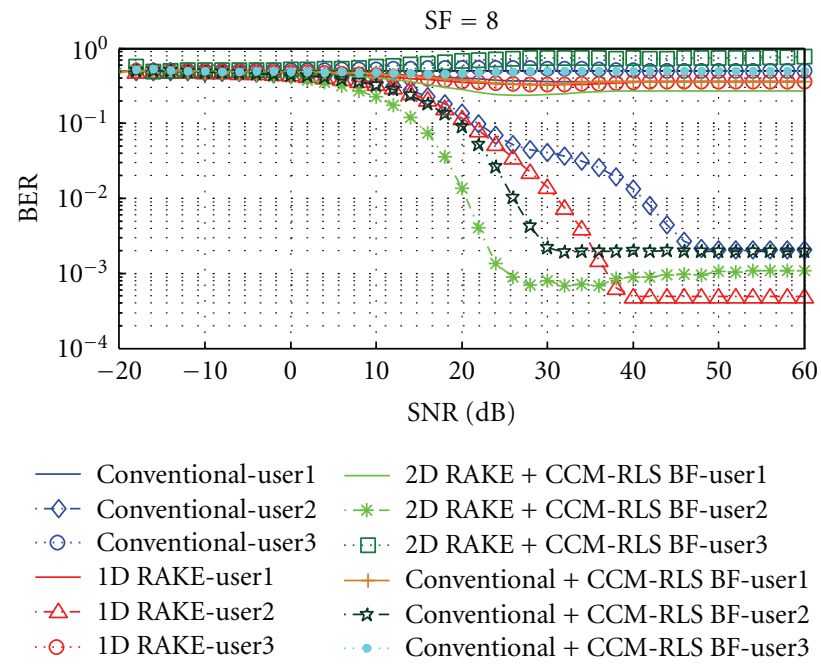

(b)

Figure 7: Performance of different conventional and proposed WCDMA receivers considering 4-element antenna array in 3-path AWGN fading channel with 3 users for (a) $\mathrm{SF}=16$, (b) $\mathrm{SF}=8$.

the BER, significantly. Also, as expected, it shows an increase in BER by increasing the number of users.

Figure 5 shows the effect of adding multipath fading to the channel. This time, the signal of each user is received with two paths by 0 and $60 T_{c}$ delays. As shown, in comparison with Figure 4, the BER in Figure 5 has been increased. In this condition, 1D-RAKE shows its advantages than conventional receiver and it is more tangible in Figure 6 by three paths with $0,55 T_{c}$, and $60 T_{c}$ delays. In all Figures, proposed receiver has better performance. The speed and slope of BER reduction while the SNR increases are more, but its complexity is also more than others.

In Figure 7, it is expressed that reducing SF is the reason for increasing BER. In other words, in constant BER, if SF increases, the capacity of the system will be enhanced. For evaluating the effect of the number of array elements in the 


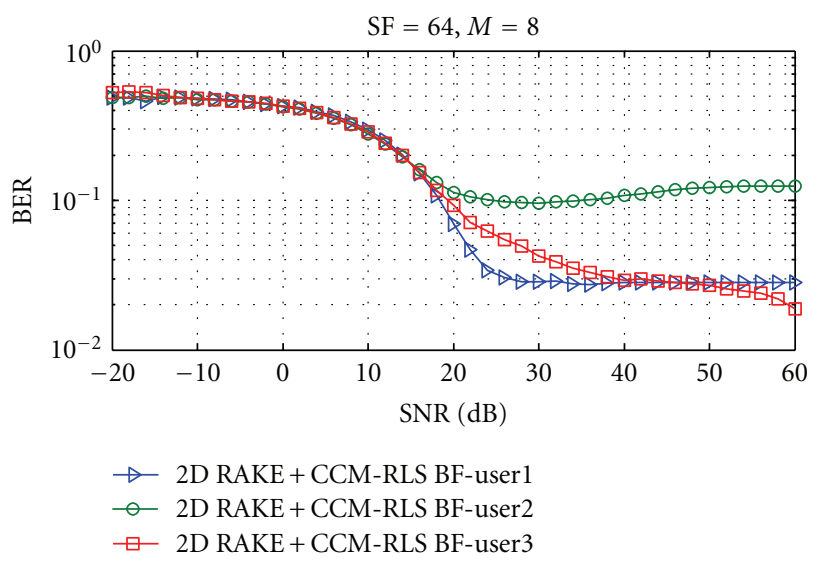

(a)

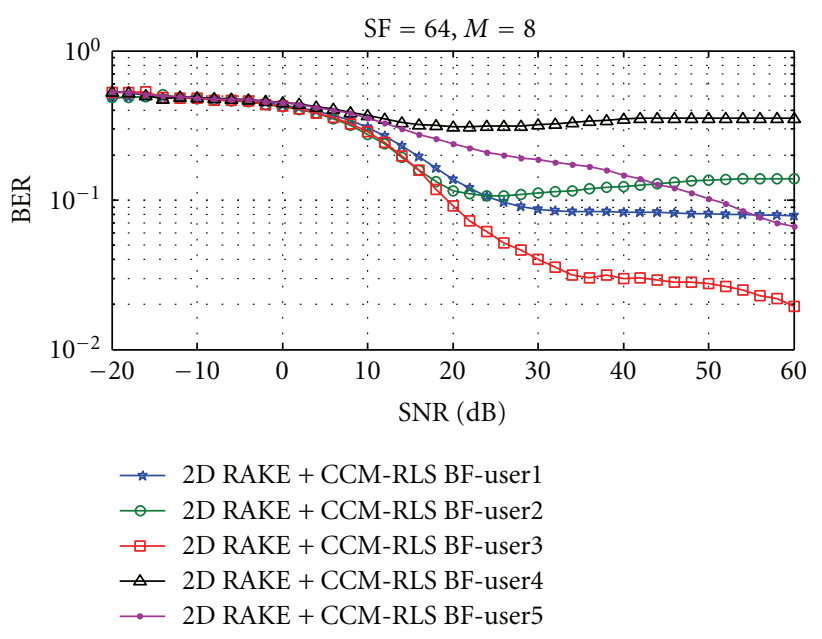

(b)

Figure 8: Performance of proposed receiver considering 8-element antenna array in 3-path AWGN fading channel for (a) 3 users, (b) 5 users.

proposed receiver, it is increased to 8 and 16. Figures 8 and 9 , show that increasing the number of array elements is the reason for increasing the slope of error reduction curve. On the other hand, complexity, cost, and occupied space will be increased. This property appears more efficiently when the number of users goes up. Simulation results show that 4 elements are enough for 3 users. If the number of users is lower than the number of array elements, system error is expected to be high because the smart antenna cannot remove the interference. Figure 10 shows this matter well.

In Figures $4-10$, after SNR $=30 \mathrm{~dB}, \mathrm{BER}$ is constant. It is due to this fact that "increasing the transmitted signal power causes increasing interference." Therefore, SINR and associated BER will be constant.

\section{Conclusion}

In this paper the performance of four receivers entitled as correlator, 1D-RAKE, correlator + CCM-RLS beamforming,

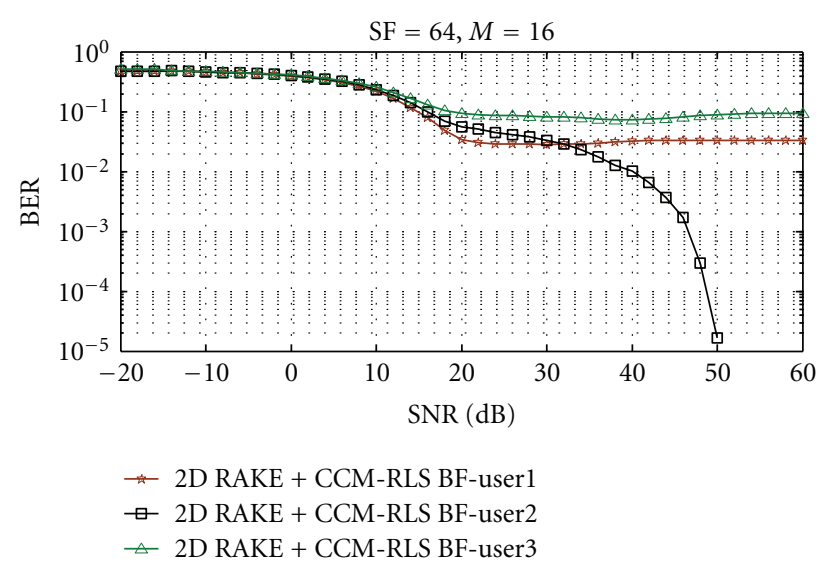

(a)

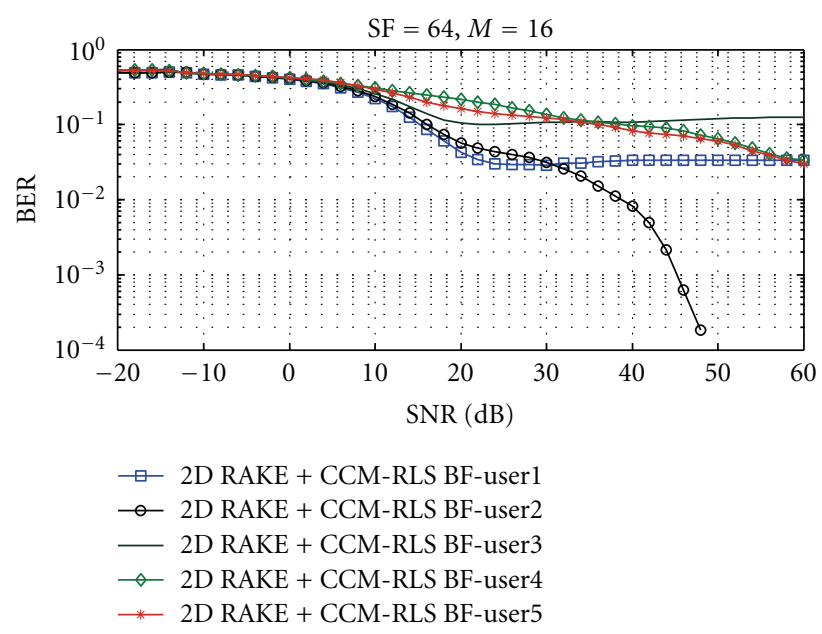

(b)

Figure 9: Performance of proposed receiver considering 16element antenna array in 3-path AWGN fading channel for (a) 3 users, (b) 5 users.

and 2D-RAKE with CCM-RLS beamforming in WCDMA system is evaluated. Correlator is not suitable for channels with multipath fading but 1D-RAKE which offers temporal diversity can reduce multipath effects. The results of correlator + CCM-RLS beamforming is almost similar to $1 \mathrm{D}$-RAKE, and according to its complexity, it is not acceptable. But 2D-RAKE with CCM-RLS beamforming, proposed in this investigation, by using advantages of both spatial and temporal diversities, shows better performance in multipath fading AWGN channels. Also the BER can be reduced to an acceptable level by increasing the number of array elements that is more understandable when the number of users increases. The only problem of the proposed receiver seems to be its complexity and cost value which will be increased by the number of users. In contrast, it should be noted that, for higher number of users, MAI will be increased and conventional receivers cannot remove interferences and are not able to offer required capacity and quality. 


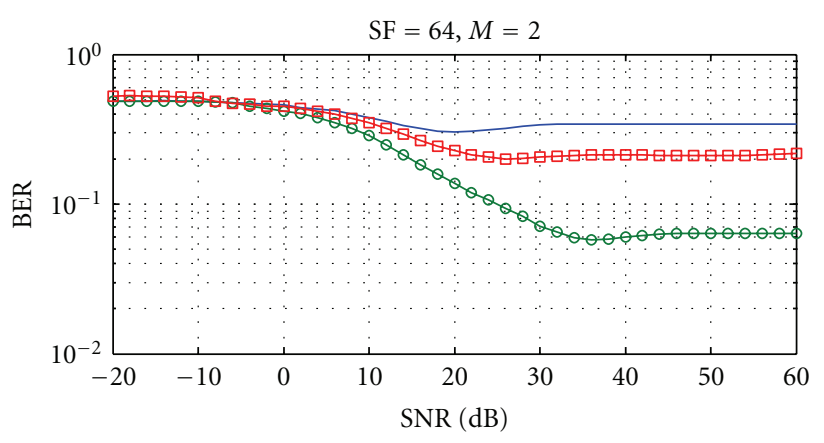

- 2D RAKE + CCM-RLS BF-user1

$\rightarrow$ 2D RAKE + CCM-RLS BF-user2

$\rightarrow$ 2D RAKE + CCM-RLS BF-user3

(a)

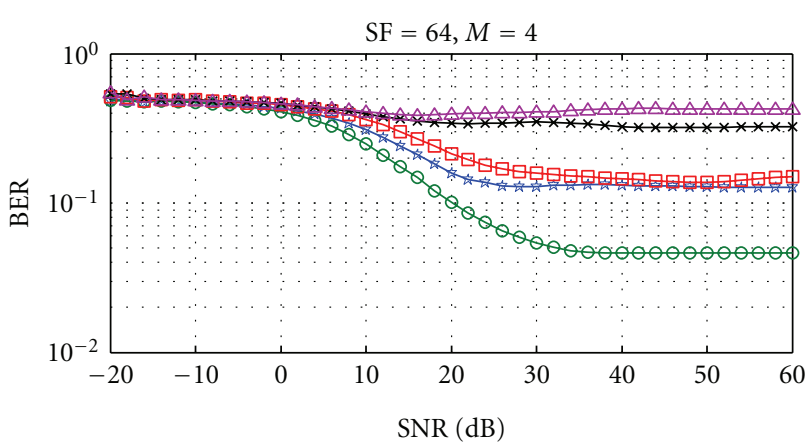

ش 2D RAKE + CCM-RLS BF-user1

- 2D RAKE + CCM-RLS BF-user2

$\square$ 2D RAKE + CCM-RLS BF-user3

$*$ 2D RAKE + CCM-RLS BF-user4

$\triangle$ 2D RAKE + CCM-RLS BF-user5

(b)

FIgURE 10: Performance of proposed receiver in 3-path AWGN fading channel and $\mathrm{SF}=64$ for (a) 2-element antenna array considering 3 users, (b) 4-element antenna array considering 5 users.

\section{Acknowledgment}

The authors would like to thank anonymous reviewers of International Journal of Antennas and Propagation (IJAP) for their careful reviews of the paper. Their comments have certainly improved the quality of this paper.

\section{References}

[1] H. Zhang, WCDMA simulator with smart antennas, M.S. thesis, Helsinki University of Technology, 2002.

[2] S. Durrani, Investigations into smart antennas for CDMA wireless systems, Ph.D. thesis, Queensland University, 2004.

[3] I. Stevanović, A. Skrivervik, and J. R. Mosig, "Smart antenna systems for mobile communications," Final Report, Ecole Polytechnique Fédérale de Lausanne, 2003.

[4] G. S. Biradar, S. N. Merchant, and U. B. Desai, "Chip equalized adaptive rake receiver for DS-CDMA UWB systems," IETE Journal of Research, vol. 56, no. 2, pp. 94-101, 2010.
[5] A. Trivedi and D. K. Mehra, "Convolutionally coded adaptive space-time MMSE receiver for W-CDMA systems in multipath channels," IETE Journal of Research, vol. 53, no. 2, pp. 137-151, 2007.

[6] T. L. Fulghum, D. A. Cairns, C. Cozzo, Y. P. E. Wang, and G. E. Bottomley, "Adaptive generalized rake reception in DS-CDMA systems," IEEE Transactions on Wireless Communications, vol. 8, no. 7, pp. 3464-3474, 2009.

[7] Y. Dai, G. Xue, T. Le-Ngoc, and J. Weng, "Combined adaptive interference cancellation with antenna array for CDMA systems," in Proceedings of the IEEE Global Telecommunication Conference (GLOBECOM '00), vol. 1, pp. 143-146, December 2000.

[8] B. S. Paul and R. Bhattacharjee, "Time and angle of arrival statistics of mobile-to-mobile communication channel employing dual annular strip model," IETE Journal of Research, vol. 56, no. 6, pp. 327-332, 2010.

[9] M. Dosaranian-Moghadam, H. Bakhshi, G. Dadashzadeh, and M. Godarzvand-Chegini, "Joint base station assignment, power control error, and adaptive beamforming for DSCDMA cellular systems in multipath fading channels," in Proceedings of the IEEE Global Mobile Congress (GMC '10), pp. 1-7, October 2010.

[10] J. Chen, J. Wang, P. Zhang, J. Yuan, and X. Shan, "An orthogonal projection based blind beamformer for DSCDMA systems," in Proceedings of the 5th IEEE Sensor Array and Multichannel Signal Processing Workshop (SAM '08), pp. 37-40, July 2008.

[11] J. G. Proakis, Digital Communications, McGraw-Hill, New York, NY, USA, 4th edition, 2001.

[12] A. J. Al-Joubory, F. M. Al-Naima, and S. S. Raouf, "WCDMA receiver structure with smart antennas system," in Proceedings of the International Conference on Computer and Communication Engineering (ICCCE '08), pp. 911-914, May 2008.

[13] A. M. Ahmed, J. Elrab, and N. S. Kamel, "Performance analysis of non-blind adaptive beamforming techniques for (3G) WCDMA uplink," in Proceedings of the International Conference on Computer and Communication Engineering (ICCCE '08), pp. 483-488, May 2008.

[14] C. H. Hu, S. Q. Lo, and Y. X. Tang, "Impact of antenna array on performance of parallel interference cancellation for WCDMA systems," in Proceedings of the International Conference on Networks Security, Wireless Communications and Trusted Computing (NSWCTC'09), pp. 657-661, April 2009.

[15] R. Suleesathira and J. Jindapun, "Combined adaptive antenna array and RAKE receiver," in Proceedings of the 10th IEEE International Symposium on Consumer Electronics (ISCE '06), pp. 1-5, September 2006.

[16] M. A. Akbar, H. B. Tila, M. Z. Khalid, and M. A. Ajaz, "Bit error rate improvement using ESPRIT based beamforming and RAKE receiver," in Proceedings of the 13th IEEE International Multitopic Conference (INMIC '09), pp. 1-6, December 2009.

[17] J. Saxena, C. S. Rai, and P. K. Bansal, "ICA based blind multi user detection for DS-CDMA systems," IETE Journal of Research, vol. 53, no. 5, pp. 417-423, 2007.

[18] K. S. Gilhousen, I. M. Jacobs, R. Padovani, A. J. Viterbi, L. A. Weaver, and C. E. Wheatley, "On the capacity of a cellular CDMA system," IEEE Transactions on Vehicular Technology, vol. 40, no. 2, pp. 303-312, 1991.

[19] R. C. de Lamare and R. Sampaio-Neto, "Blind adaptive code-constrained constant modulus algorithms for CDMA interference suppression in multipath channels," IEEE Communications Letters, vol. 9, no. 4, pp. 334-336, 2005. 
[20] Z. Xu and P. Liu, "Code-constrained blind detection of CDMA signals in multipath channels," IEEE Signal Processing Letters, vol. 9, no. 12, pp. 389-392, 2002.

[21] S. H. Marikar, Resource management in $3 G$ systems employing smart antennas, M.S. thesis, Faculty of the Virginia Polytechnic Institute and State University, 2002.

[22] J. Míguez and L. Castedo, "A linearly constrained constant modulus approach to blind adaptive multiuser interference suppression," IEEE Communications Letters, vol. 2, pp. 16421648, 1998.

[23] R. Price and P. E. Green, "A communication technique for multipath channels," in Proceedings of the Institute of Radio Engineers (IRE '58), vol. 46, no. 3, pp. 555-570, March 1958.

[24] W. C. Y. Lee, Wireless and Cellular Telecommunications, McGraw-Hill, 3rd edition, 2006.

[25] S. Fukumoto, M. Sawahash, and F. Adachi, "Matched filterbased RAKE combiner for wideband DS-CDMA mobile radio," IEICE Transactions on Communications, vol. E81-B, no. 7, pp. 1384-1390, 1998.

[26] D. G. Manolakis, V. K. Ingle, and S. M. Kogon, Statistical and Adaptive Signal Processing, McGraw-Hill, 2005.

[27] P. Stoica and A. Nehorai, "MUSIC, maximum likelihood, and Cramer-Rao bound," IEEE Transactions on Acoustics, Speech, and Signal Processing, vol. 37, no. 5, pp. 720-741, 1989.

[28] L. Wang and R. C. de Lamare, "Constrained constant modulus RLS-based blind adaptive beamforming algorithm for smart antennas," in Proceedings of the 4th IEEE International Symposium on Wireless Communication Systems (ISWCS 07), pp. 657-661, October 2007.

[29] L. Wang, R. C. de Lamare, and Y. L. Cai, "Low-complexity adaptive step size constrained constant modulus SG algorithms for adaptive beamforming," Signal Processing, vol. 89, no. 12, pp. 2503-2513, 2009.

[30] H. Sadeghi, S. Shirvani-Moghaddam, and V. T. Vakili, "Appropriate CCM-based algorithm for adaptive antenna array beamforming," in Proceedings of the 5th International Symposium on Telecommunications (IST '10), pp. 69-75, December 2010. 

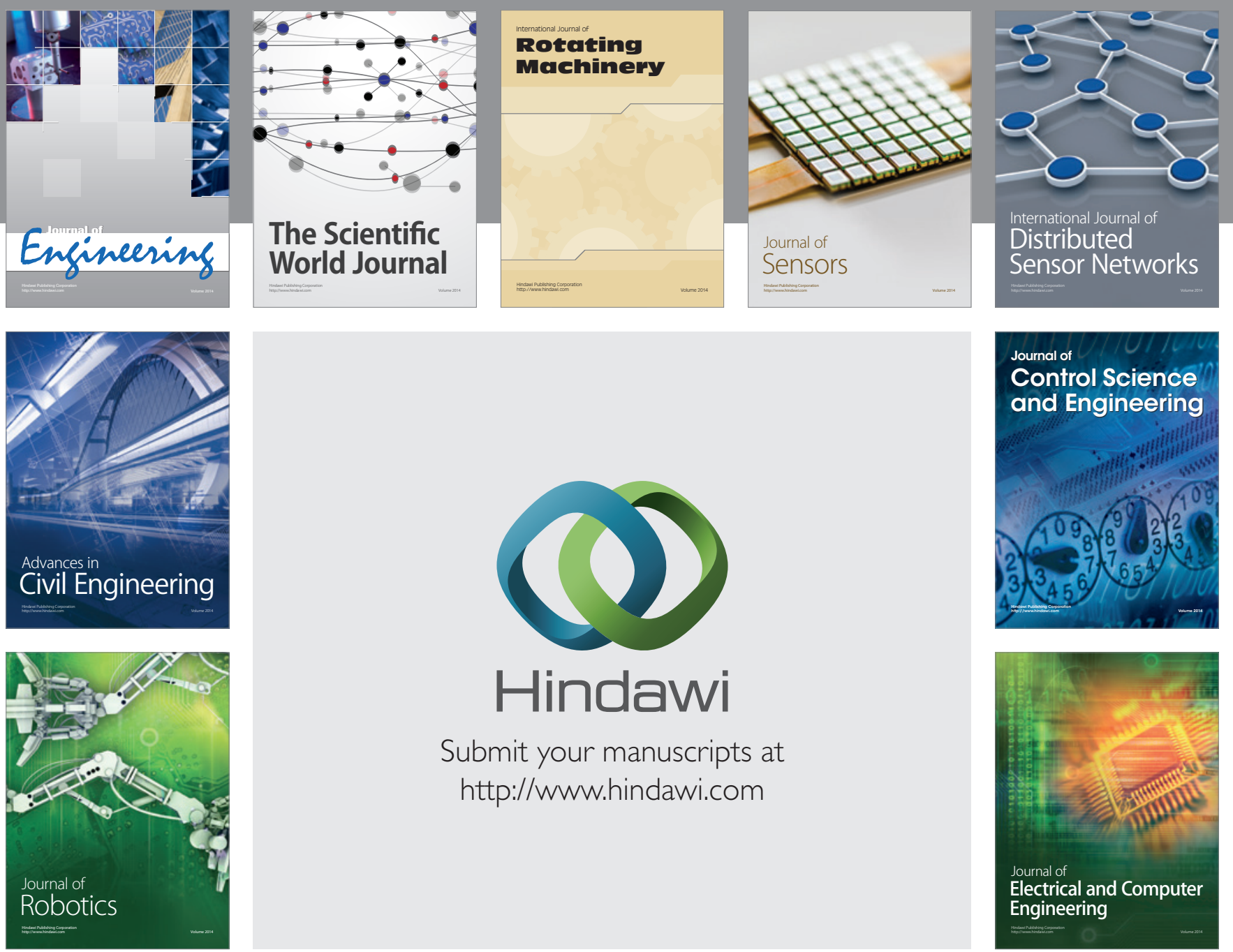

Submit your manuscripts at

http://www.hindawi.com
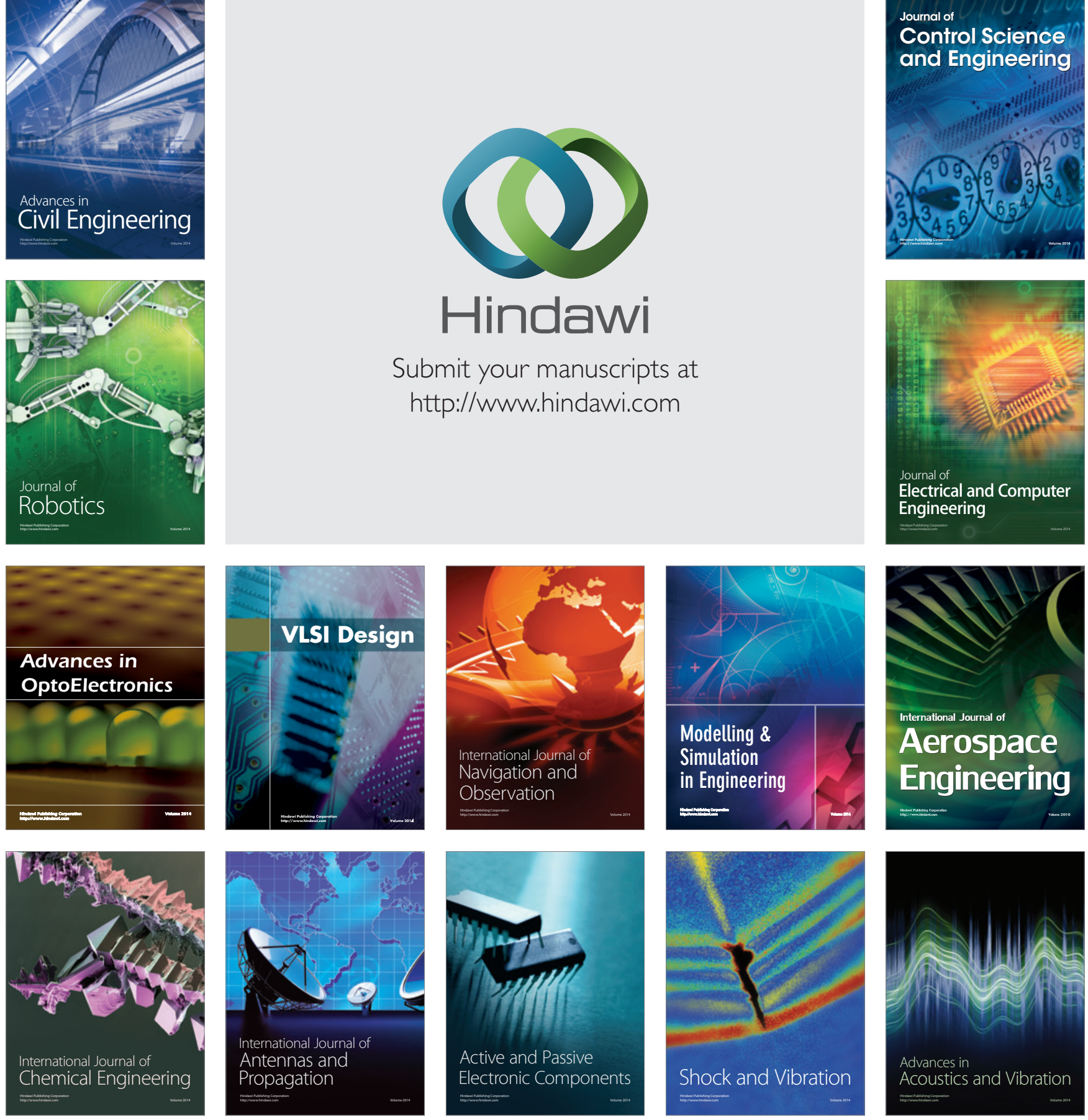